'Departamento de Salud Pública y Epidemiología, Facultad de Medicina, Universidad de los Andes. Santiago, Chile. asstudiante de Medicina Universidad de los Andes. Santiago, Chile.

bepidemióloga-Bioestadística.

Apoyo financiero: El único aporte financiero fue por parte de la Academia Científica de Estudiantes de Medicina de la Universidad de los Andes (ACEM UANDES) para cubrir el "Cobro por recepción del Manuscrito" de la Revista Médica de Chile. La ACEM UANDES no tuvo influencia en el diseño del estudio, así como tampoco en la recolección, análisis o

interpretación de los datos, ni en la preparación, revisión o aprobación del manuscrito.

Los autores declaran no tener conflictos de interés.

Recibido el 16 de mayo de 2019, aceptado el 17 de enero de 2020.

Correspondencia a: Marcelo Francisco Concha Infante Monseñor Álvaro del Portillo 12.455. Las Condes, Santiago, Chile. mfconcha1@miuandes.cl

\section{Medición de la calidad del trato al usuario tras la introducción del asistente-alumno en centros de salud familiar en Chile}

\author{
MARCELO CONCHA I. , ALAN HIRSCHBERG G. ${ }^{\text {, }}$ \\ NICOLÁS ARRAÑO R. ${ }^{a}$, MARCELA CÁRCAMO I. ${ }^{1, b}$, \\ JAIME MAÑALICH M. ${ }^{1}$
}

\section{Effect of the presence of student-assistants on the quality of care perception by ambulatory patients}

Background: The time dedicated to patients and how they are treated are crucial in the evaluation of health care quality. Medical students acting as medical assistants could improve the perception of a good quality of care among ambulatory patients. Aim: To evaluate if the presence of Student-Assistants improves the patients' perception of health care quality in ambulatory primary care. $\mathbf{P a}$ tients and Methods: Quasi-experimental exploratory study. In two health care centers, patients answered a questionnaire about their perception of how they were treated at baseline and after an intervention period. In one center, prior to the appointment of the patient with the doctor, the student interviewed patients focusing on chief complaints and registered their vital signs, orally presenting this information to the clinician. In the other center, there was no student intervention. Patients answered the questionnaire at the end of appointments. Results: At baseline 103 patients answered the questionnaire (58 in the experimental and 45 in the control center). After the intervention, 121 patients answered it (56 in the experimental and 65 in the control center). Basal scores were 6,25 and 6,06 in experimental and control center, respectively $(p=N S)$. After the intervention, the scores were 6,49 and 6,15, respectively $(p=0,01)$. Conclusions: These data support the hypothesis that the presence of a Student-Assistant could improve the perception of patients about how they are treated at primary health care centers.

(Rev Med Chile 2020; 148: 60-68)

Key words: Patient Care Team; Primary Health Care; Quality of Health Care; Students, Medical.

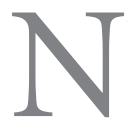
umerosa evidencia señala los beneficios de sistemas sanitarios basados en una atención primaria de salud (APS) ${ }^{1-5}$; de hecho, la Organización Panamericana de la Salud hace un llamado al potenciamiento de este modelo ${ }^{5}$.

En Chile, desde 2005 se inicia un proceso de reforma sanitaria cuyos pilares han sido la creación del Plan de Acceso Universal a Garantías Explícitas (actuales Garantías Explícitas en Salud) y el fortalecimiento de la APS ${ }^{6,7}$.

El diseño de intervenciones dirigidas al mejoramiento de la APS exige conocer los indicadores y factores asociados a la calidad de la atención de salud. En efecto, se han propuesto y evaluado una amplia variedad de indicadores de calidad en APS y cuidados ambulatorios ${ }^{8-10}$. 
Una de las dimensiones que componen la calidad es la satisfacción usuaria, siendo el trato recibido uno de los subcomponentes de esta ${ }^{11}$. Tal es la importancia de la relación con los usuarios, que una de las medidas implementadas en Chile para el mejoramiento de la calidad de atención en la APS municipal ha sido el ordenamiento del pago de un bono anual a los funcionarios de dichos establecimientos, según la ubicación de su centro en un ranking de calidad de trato a usuario realizado durante el año en cuestión (Ley 20.645) ${ }^{12}$.

Por otro lado, el tiempo disponible para la atención de salud también se ha reconocido como uno de los factores determinantes de su calidad ${ }^{13-15}$.

Así, se han descrito diversas propuestas sobre la organización de recursos para cubrir las demandas de atención en APS. Por ejemplo, Rosser et al. (2011) describen la experiencia de la implementación, en Ontario, Canadá, del modelo Family Health Team ${ }^{16}$. Por su parte, Norful et al. (2017) hacen una revisión sobre la inclusión de las Registred Nurses en la APS ${ }^{17}$. Asimismo, otros trabajos se refieren a la incorporación de los medical assistants (asistentes médicos) en los equipos de atención de este nivel ${ }^{18-21}$.

En el modelo denominado teamlets (equipos de trabajo), descrito por Bodenheimer en Estados Unidos de Norteamérica, la atención de los pacientes queda a cargo de pequeños equipos de salud conformados por un clínico y sus ayudantes, denominados health coaches (consejeros de salud), cuyo rol puede ser asumido por diferentes profesionales entrenados para tal efecto ${ }^{22,23}$.

Bodenheimer propone la estructuración de la atención en las fases de preconsulta, consulta, postconsulta y cuidados interconsulta. En la preconsulta, el health coach lleva a cabo una historia clínica básica y realiza algunas mediciones simples. Durante la consulta, el clínico toma conocimiento del caso y profundiza la anamnesis mientras el health coach le asiste en diversas labores. En la postconsulta, el health coach resuelve dudas del paciente y refuerza las indicaciones del clínico. En la última fase, el health coach asume el rol de seguimiento del paciente, sirviendo de intermediario entre este y el clínico.

Teniendo en cuenta lo expuesto, parece necesario explorar nuevas iniciativas que contribuyan al mejoramiento de la APS. Así, considerando la existencia de alianzas docente-asistencial entre universidades y centros de salud ${ }^{24}$, junto con la flexibilidad y oportunidades que ofrece el modelo de teamlets, parece factible que un estudiante de medicina pueda asumir un rol similar al de un health coach (asistente-alumno) y ser un aporte en la APS. En efecto, el objetivo principal de este estudio fue evaluar si la introducción del asistente-alumno mejora la percepción de calidad del trato de pacientes en consultas de morbilidad en un centro de salud familiar (CESFAM). El objetivo secundario fue conocer la opinión médica sobre esta iniciativa a través de una encuesta.

\section{Pacientes y Método}

\section{Diseño}

Estudio exploratorio cuasiexperimental. Se realizaron mediciones pre y postintervención en un centro experimental y un centro control (Figura 1) durante diciembre de 2017, seleccionados por conveniencia.

\section{Criterios de inclusión y exclusión}

Se consideraron centros de una misma comuna (Maipú), con una población inscrita validada por FONASA mayor a 30.000 personas. Se excluyeron centros ubicados en el tramo mejor evaluado del ranking de trato a usuario 2016 (Figura 1).

En base a reunión sostenida con autoridades de la salud municipal de Maipú, se escogió el CESFAM Dr. Carlos Godoy como centro experimental y el CESFAM Presidenta Michelle Bachelet como centro control. El primero posee 31.516 pacientes inscritos y el segundo $33.098^{25}$, ubicándose, respectivamente, en la zona centro-poniente y central del área urbana de la comuna de Maipú ${ }^{26}$.

Se incluyeron pacientes hispanoparlantes entre 30 y 70 años que asistían a consultas de morbilidad (Tabla 1) (Barra E. Investigación UANDES. 2018. Trabajo no publicado). No se consideraron criterios de exclusión.

\section{Método}

Durante la primera semana del estudio se hizo una medición basal de calidad del trato en ambos centros, en forma simultánea (Figura 1). Para ello, se utilizó el "Cuestionario de calidad del trato usuario en los establecimientos de APS (cuestionario TU-APS)". Dicho cuestionario fue validado previamente en el año 2012 ( $\alpha$ de Cronbach $=0,94)^{27}$ y se utiliza anualmente en la elaboración del ranking de trato a usuario mencionado previamente. Se compone de 33 preguntas, 


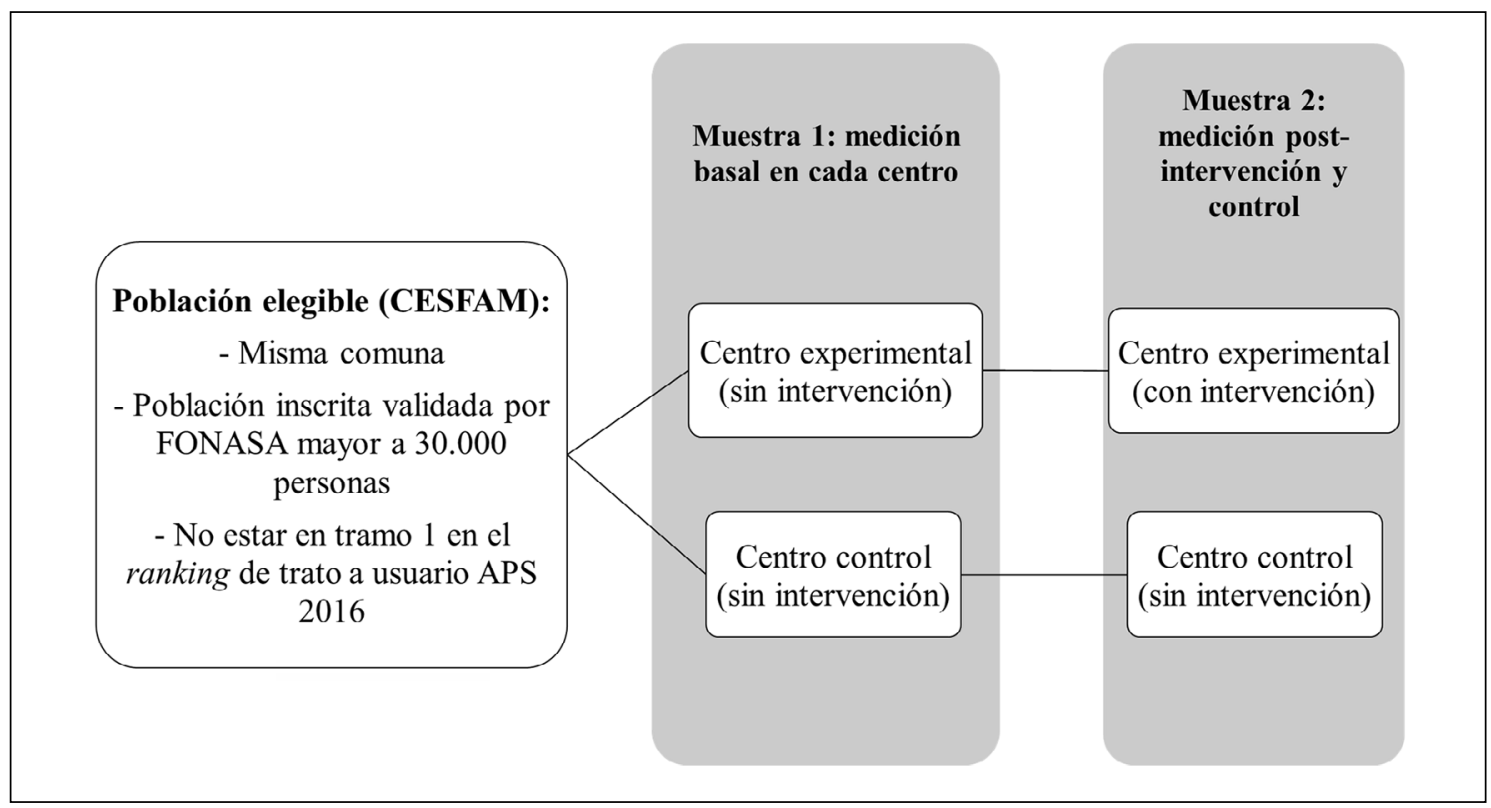

Figura 1. Metodología. Se realizó una medición basal en ambos centros utilizando la encuesta TU-APS. Posteriormente, se introdujo al asistente-alumno en uno de los centros (experimental), mientras que el otro no fue intervenido (control). Se aplicó el mismo instrumento para medir los resultados postintervención y control.

Tabla 1. Población inscrita validada entre 30 y 70 años cumplidos

\begin{tabular}{|lcccc|}
\hline Centro & \multicolumn{2}{c}{ Experimental } & \multicolumn{2}{c|}{ Control } \\
& $\mathbf{n}$ & \% & \multicolumn{1}{c|}{ n } & \% \\
Hombres & 5.891 & 40 & 5.905 & 36 \\
Mujeres & 8.919 & 60 & 10.350 & 64 \\
Total & 14.810 & 100 & 16.255 & 100 \\
\hline
\end{tabular}

n: frecuencia absoluta; \%: frecuencia relativa.

en 24 de ellas, el paciente evalúa con escala de 1 a 7 (siendo 1 la peor y 7 la mejor calificación) los distintos estamentos implicados en la atención: administrativo, técnico-parámedico y profesional $^{28}$. La encuesta se aplicó luego de la consulta con el médico. Esta última contaba con una duración de 15 minutos por paciente en ambos centros.

Se consultó a los pacientes si querían o no participar del estudio. Si aceptaban participar, se procedía a la lectura y firma del consentimiento informado previo a la consulta y a la aplicación del cuestionario TU-APS posterior a la misma. Esto estuvo a cargo de cuatro estudiantes de enfermería de tercer año que rotaron continuamente durante todo el estudio entre los dos centros.
Durante las dos semanas siguientes, tres estudiantes de medicina de cuarto año participaron como asistente-alumno en el centro experimental (Figura 1). Ellos entrevistaron a los pacientes antes del ingreso a la consulta, focalizándose en la anamnesis próxima y registro de signos vitales (presión arterial, pulso y temperatura) en un box de atención privado. Se desarrolló una pauta de entrevista con información relevante a obtener ante algunos síntomas frecuentes que los alumnos utilizaron como material de apoyo impreso.

Posteriormente, los pacientes eran conducidos a la sala de espera, donde permanecían hasta el llamado del médico. Durante esta espera, los alumnos no mantuvieron contacto con los pacientes.

$\mathrm{Al}$ momento del llamado, el asistente-alumno ingresaba al box junto con el paciente, donde presentaba oralmente el caso al médico. Luego, el estudiante salía del box para permitir que el profesional desarrollara la consulta en forma habitual. Finalizada la consulta, se aplicó el cuestionario TU-APS.

Finalizado el estudio, los médicos que interactuaron con los estudiantes registraron su opinión en una encuesta anónima. Esta fue diseñada por los autores y se compone de 4 preguntas cerradas en las 
que se pide indicar el nivel de acuerdo o desacuerdo respecto a una afirmación y 2 preguntas abiertas.

\section{Tamaño de muestra}

Se consideraron los resultados del "Estudio Nacional de Evaluación de Percepción del Trato a Usuarios en Establecimientos de Salud Municipal del Sistema Público de Salud" (2013) ${ }^{28}$. Se hizo un cálculo en base a la peor y mejor media ( $\mathrm{DE} \pm$ ): $6,03 \pm 0,99$ y $6,60 \pm 0,65$, con una potencia de $80 \%$ y un nivel de significación de 5\%, obteniéndose 35 pacientes por grupo. Se consideró un porcentaje de pérdida de $10 \%$, considerando 40 pacientes por grupo $(\mathrm{n}=80)$.

\section{Análisis estadístico}

Se realizó estadística descriptiva según tipo y escala de variable. Los resultados se reportaron en tablas.

El cálculo del promedio global del cuestionario se hizo sobre un total de 24 preguntas. En pacientes que no fueron atendidos por el estamento técnico-paramédico, el promedio se calculó sobre un total de 18 preguntas. En encuestas con notas faltantes por omisión del paciente o del encuestador, el promedio se calculó sobre un total de 24 preguntas.

La comparación de los promedios de notas entre los centros se realizó mediante la prueba t-Student. Los promedios se reportaron con sus respectivos intervalos de confianza (IC 95\%). Los análisis se realizaron con STATA 14.0 (Licencia Universidad de los Andes). Se utilizó un nivel de significación de $5 \%$.

Para presentar las opiniones de los médicos, se hicieron gráficos y una tabla con Microsoft ${ }^{\circ}$ Excel $^{\oplus}$ para Office 365. (Microsoft Corporation, Redmond, WA, USA).

\section{Aspectos éticos}

Se respetaron las normas éticas establecidas en la Declaración de Helsinki y se obtuvo la aprobación del Comité Ético Científico de la Universidad de los Andes (FOLIO: CEC 201736). Además, se contó con el permiso del jefe de Gestión Sanitaria de la Municipalidad de Maipú y los directores de ambos centros. Toda la información se obtuvo a partir de la entrevista con los pacientes; ningún estudiante tuvo acceso a la ficha de estos, ni tampoco opinión sobre el diagnóstico o tratamiento.

\section{Resultados}

Participó un total de 224 pacientes. En los 4 grupos hubo mayor porcentaje de mujeres y el promedio de edad, para el grupo pre y postintervención, se mantuvo entre 55,54 (DE $\pm 8,99)$ y 57,32 (DE $\pm 8,64$ ) años. En los 4 grupos, el tramo FONASA B fue el que tuvo mayor proporción de pacientes (Tabla 2).

Tabla 2. Caracterización de las muestras

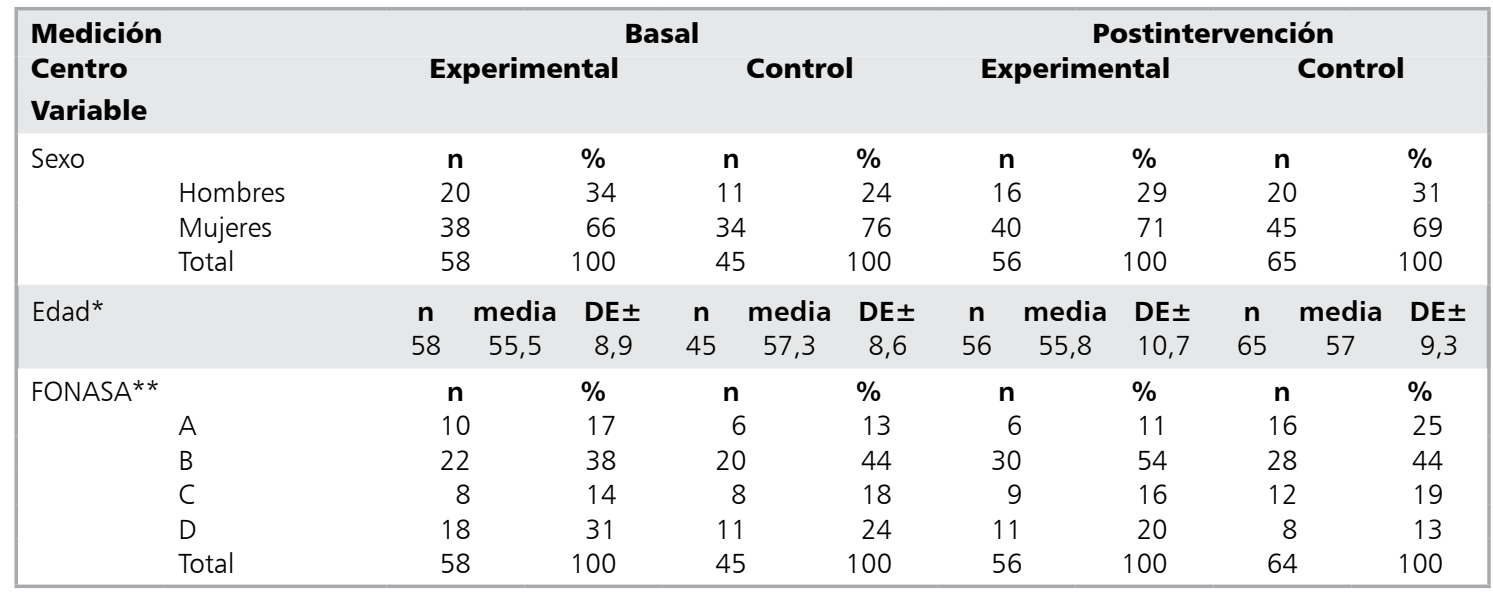

*Edad en años. **En la medición postintervención, un paciente del centro control pertenecía al Programa de Reparación y Atención Integral de Salud (PRAIS), lo que explica que la sumatoria de pacientes según tramo FONASA sea 64 y 65; n: frecuencia absoluta; \%: frecuencia relativa; DE \pm : desviación estándar. 
En la medición basal, el promedio global del cuestionario en el centro experimental fue 6,25 (IC 95\% 6,08-6,42) y 6,06 (IC 95\% 5,79-6,33) en el centro control, no habiendo diferencias estadísticamente significativas $(\mathrm{p}=0,2)$. Luego de la intervención, los promedios mostraron diferencias, con 6,49 (IC 95\% 6,31-6,68) en el centro experimental contra 6,15 (IC 95\% 5,95-6,35) en el centro control $(p=0,01)$. No hubo diferencias estadísticas entre la medición basal y postintervención de cada centro (Tabla 3 ).
La evaluación general del trato tuvo un comportamiento similar al promedio global del cuestionario (Tabla 4).

En el centro experimental, la evaluación del trato del profesional subió de 6,36 (IC 95\% 6,16,63 ) basal a 6,75 (IC 95\% 6,51-6,99) postintervención $(\mathrm{p}=0,03)$. El centro control obtuvo 6,22 (IC 95\% 5,87-6,58) en la primera medición y 6,29 (IC 95\% 6,01-6,58) en la segunda. Postintervención, hubo diferencias entre los centros $(6,75$ contra $6,29, \mathrm{p}=0,02)($ Tabla 5).

Tabla 3. Promedio global del cuestionario TU-APS

\begin{tabular}{|c|c|c|c|c|c|c|c|c|c|}
\hline \multirow[b]{3}{*}{ Centro } & \multirow[b]{3}{*}{$\mathbf{n}$} & \multirow[b]{3}{*}{ media } & \multicolumn{6}{|c|}{ Medición } & \multirow{3}{*}{ p } \\
\hline & & & \multirow{2}{*}{$\begin{array}{c}\text { Basal } \\
\text { IC } 95 \%\end{array}$} & & & \multicolumn{3}{|c|}{ Postintervención } & \\
\hline & & & & DE \pm & $\mathbf{n}$ & media & IC $95 \%$ & DE \pm & \\
\hline Experimental & 58 & 6,253 & $(6,084-6,421)$ & 0,640 & 56 & 6,494 & $(6,308-6,680)$ & 0,693 & 0,056 \\
\hline Control & 45 & 6,057 & $(5,788-6,327)$ & 0,897 & 65 & 6,150 & $(5,948-6,352)$ & 0,814 & 0,573 \\
\hline$p^{\prime}$ & & & 0,199 & & & & 0,014 & & \\
\hline
\end{tabular}

p: test t-Student para 2 promedios con varianza similar entre promedio basal y postintervención, en cada centro; $p^{\prime}$ : test t-Student para 2 promedios con varianza similar entre el promedio del centro experimental y control, en cada medición; n: número de pacientes; IC 95\%: intervalo de confianza al 95\%; DE 土: desviación estándar.

Tabla 4. Evaluación general del trato

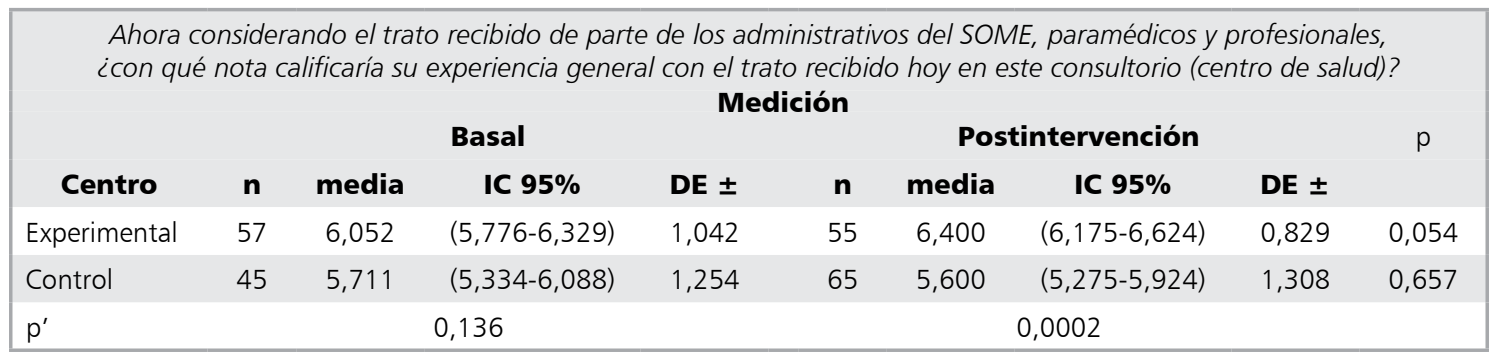

p: test t-Student para 2 promedios con varianza similar entre la nota basal y postintervención, en cada centro; p': test t-Student para 2 promedios con varianza similar entre la nota del centro experimental y control, en cada medición; n: número de pacientes; IC 95\%: intervalo de confianza al 95\%; DE 土: desviación estándar.

\section{Tabla 5. Evaluación del trato del profesional}

\begin{tabular}{|c|c|c|c|c|c|c|c|c|c|}
\hline \multirow[b]{3}{*}{ Centro } & \multirow{2}{*}{\multicolumn{8}{|c|}{ Postintervención }} & \multirow{3}{*}{$\mathrm{p}$} \\
\hline & & & & & & & & & \\
\hline & $\mathbf{n}$ & media & IC $95 \%$ & DE \pm & $\mathbf{n}$ & media & IC $95 \%$ & DE \pm & \\
\hline Experimental & 58 & 6,362 & $(6,098-6,625)$ & 1,003 & 56 & 6,750 & $(6,509-6,990)$ & 0,899 & 0,032 \\
\hline Control & 45 & 6,222 & $(5,866-6,578)$ & 1,184 & 65 & 6,292 & $(6,009-6,575)$ & 1,141 & 0,755 \\
\hline$p^{\prime}$ & & & 0,518 & & & & 0,017 & & \\
\hline
\end{tabular}

p: test t-Student para 2 promedios con varianza similar entre la nota basal y postintervención, en cada centro; p': test t-Student para 2 promedios con varianza similar entre la nota del centro experimental y control, en cada medición; n: número de pacientes; IC 95\%: intervalo de confianza al 95\%; DE 土: desviación estándar. 


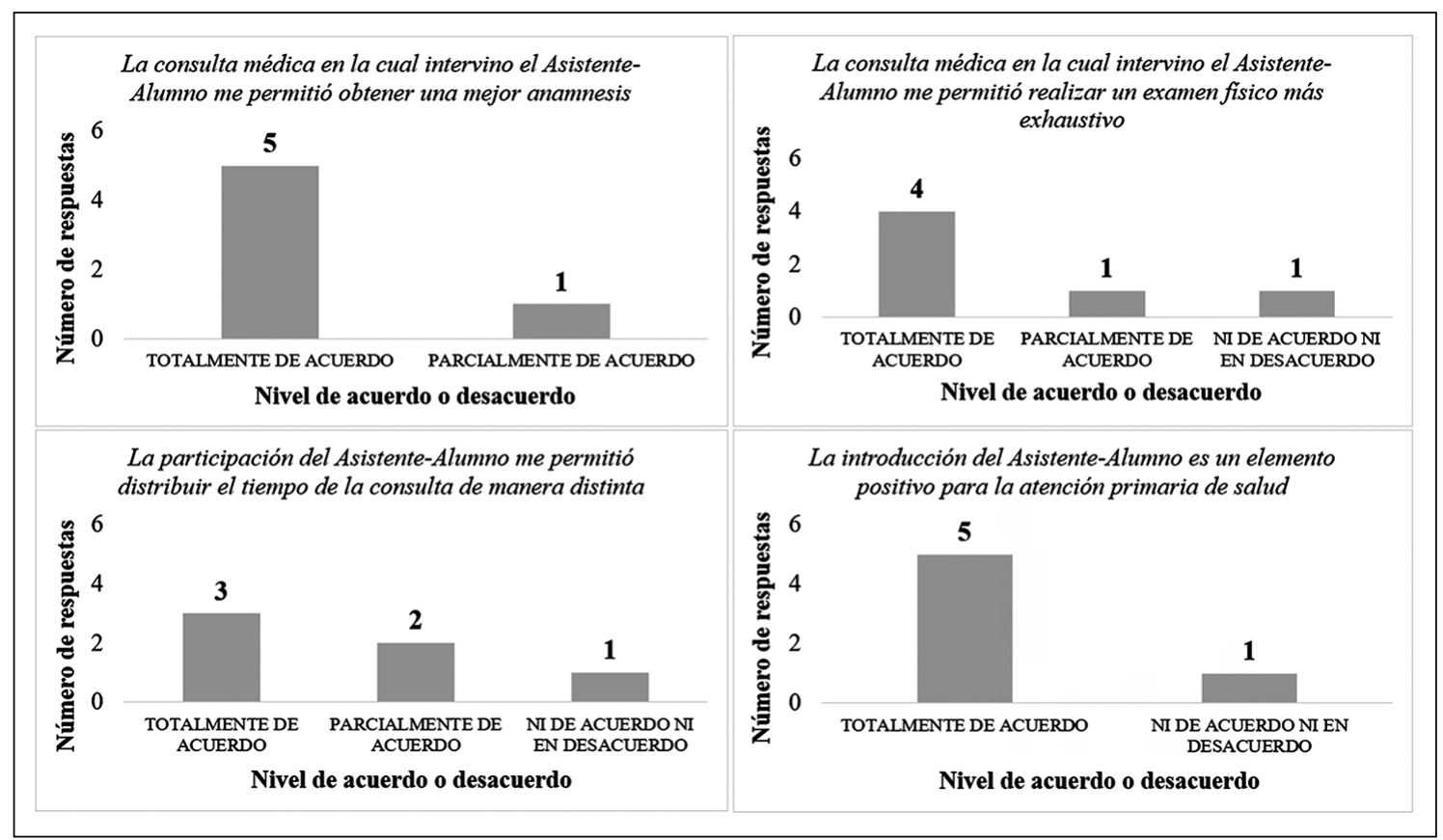

Figura 2. Opinión de los médicos acerca del asistente-alumno. Se muestra el nivel de acuerdo o desacuerdo de 6 médicos encuestados acerca de 4 afirmaciones en relación con la participación del asistente-alumno.

\section{Tabla 6. Opiniones de los médicos acerca del asistente-alumno}

\section{¿Qué cambió en la distribución del tiempo de consulta?}

"Se obtienen antecedentes relevantes previo al ingreso del paciente. Se logra anamnesis dirigida en menos tiempo, sin perder datos relevantes y se cuenta con más tiempo para examen físico acucioso"

"Se mejoró calidad de atención por la centralización de motivos de consulta y control del paciente"

"Me permitió realizar un examen físico más detallado y orientado a la patología según el caso, ya que el "asistente-alumno" adelanta parte del trabajo"

"Acorta el tiempo para llegar al motivo de consulta central. El paciente se siente doblemente acogido"
La Figura 2 y la Tabla 6 resumen los resultados de la encuesta aplicada a los médicos.

\section{Discusión}

$\mathrm{Al}$ analizar el trato en forma general, ya sea atendiendo al promedio global del cuestionario o a la pregunta específica que evalúa este ítem, los resultados fueron similares y mostraron una

\section{Sugerencias}

"Acceso a ficha electrónica. Registro previo de datos en ficha para optimizar tiempo, de lo contrario no mejora la distribución del tiempo"

"Mantener (dentro de lo posible) la presencia del "asistente-alumno" previo a la consulta de morbilidad"

"Ninguna en lo particular. Excelente iniciativa en la formación de los futuros médicos, así se incorporan para mayor sensibilidad y trato social" 
Es de especial interés lo sucedido con la evaluación del trato del profesional, pues, además de haber diferencias estadísticamente significativas entre los centros luego de la intervención, el aumento en la calificación del centro experimental también fue estadísticamente significativo respecto de su basal.

Estas mejorías podrían ser explicadas, por un lado, debido a que la interacción paciente-alumno haya sido una experiencia positiva en sí misma, lo que ha sido descrito en otros trabajos que estudiaron este tema ${ }^{29,30}$. Por ejemplo, en un contexto de APS, Prislin et al. (2001) reportaron que los pacientes consideraron que su participación en la formación de los alumnos fue una experiencia agradable, y manifestaron que la participación de estos mejoró la calidad de la atención recibida ${ }^{29}$. Asimismo, aunque en un contexto de pacientes hospitalizados en Chile, Beca et al. (2006) reportaron que estos se sintieron cómodos al ser escuchados por estudiantes ${ }^{30}$. Por otro lado, según las opiniones de los médicos, el aporte del asistente-alumno pareciera haberles dado la posibilidad de abordar la consulta de mejor manera, lo que también pudo haber contribuido a la mejora en el trato. Así, sería razonable pensar que las dos explicaciones recién discutidas tengan un efecto sinérgico, dando cuenta de los resultados.

De las opiniones manifestadas se deduce que los médicos percibieron al asistente-alumno como un elemento positivo, que podía aportarles tanto a ellos en su labor profesional como a los pacientes. Esta recepción positiva podría significar que el asistente-alumno sea considerado como un elemento de valor en el equipo de salud, lo que parece concordar con el reconocimiento del trabajo en equipo como un elemento central para enfrentar la atención de salud en APS ${ }^{23,31-36}$.

En el estudio chileno de M. Parada-Lezcano et al. (2016), los estudiantes de medicina manifestaron un distanciamiento entre los contenidos enseñados (centrados en especialidades hospitalarias) y el futuro ejercicio profesional, lo que les generaba inseguridad sobre su capacidad de desempeño en APS. También se pesquisó la idea de que el alumno se contactaba tardíamente con la APS durante su formación, centrándose sus prácticas mayoritariamente en los hospitales ${ }^{37}$.

Ahora bien, así como en la revisión de Dornan et al. (2006) se reportan múltiples beneficios para los estudiantes de su incorporación temprana a la práctica clínica ${ }^{38}$, sería razonable hipotetizar que un enfrentamiento temprano del estudiante con la APS, por ejemplo, bajo el rol de asistente-alumno, podría ayudar a que estos adquieran confianza sobre cómo desempeñarse en este contexto. A este respecto, es interesante revisar la experiencia internacional de los clerkships (estudiantes en práctica), rotaciones que tienen por objetivo el acercamiento de estudiantes de pregrado a la APS $^{39-41}$, dando cuenta de la factibilidad de la incorporación de los alumnos a este nivel de atención.

Como limitantes de este trabajo hay que considerar el diseño no aleatorizado, lo que no permite demostrar una real eficacia de la intervención. Además, cabe señalar la falta de control sobre otras variables de interés, por ejemplo, la falta de inclusión de un número homogéneo de pacientes de los distintos sectores de atención en los que se estructuran los centros. Otro elemento a considerar es la época de realización (verano), ya que podría representar un escenario distinto a lo esperable en otros períodos (invierno). Por último, estos resultados corresponden a una experiencia aislada, por lo que su validez externa es escasa. Esto plantea la necesidad de más estudios para evaluar si los resultados se replican en otros contextos.

Una fortaleza de este trabajo es que participaron pacientes afiliados a FONASA, sistema que acoge a $78 \%$ de la población ${ }^{42}$. Por otro lado, es interesante el enfoque del aporte desde el alumno hacia el paciente y el profesional. Finalmente, la iniciativa es fácilmente replicable.

Debido a que este estudio se ha centrado solo en la percepción de los pacientes y, secundariamente, en la opinión de los profesionales, sería atractivo contar con un trabajo que evalúe la percepción de los estudiantes bajo el rol de asistente-alumno.

\section{Conclusión}

La presencia del asistente-alumno mostró una diferencia favorable, estadísticamente significativa, en el promedio global del cuestionario de trato a usuario entre el centro intervenido y control. Este trabajo es un paso en la consideración del estudiante de medicina como un factor asociado a la calidad de atención en APS, posicionándolo como un posible actor de cambio de la salud pública chilena. 
Agradecimientos: A los directores de los centros Sra. Tamara Riquelme Becerra y Sr. Gustavo Cifuentes, así como a los equipos de salud por abrir sus puertas. A las estudiantes de enfermería de la Universidad de los Andes Camila Paredes, Nicole Matus, Gabriela Figueroa, Catalina Chavarría por su ayuda y compromiso. A los doctores Martín Plaza G. y Andrés Glasinovic P. por su asesoría en la escritura de este artículo.

\section{Referencias}

1. Starfield B. Is primary care essential? Lancet 1994; 344 (8930): 1129-33.

2. Starfield B, Shi L. Policy relevant determinants of health: an international perspective. Health Policy 2002; 60 (3): 201-18.

3. Starfield B. Primary care: an increasingly important contributor to effectiveness, equity, and efficiency of health services. SESPAS report 2012. Gac Sanit 2012; 26: 20-6.

4. Macinko J, Starfield B, Shi L. The Contribution of Primary Care Systems to Health Outcomes within Organization for Economic Cooperation and Development (OECD) Countries, 1970-1998. Health Serv Res 2003; 38 (3): 831-65.

5. Macinko JA, Montenegro Arriagada H, Nebot C, Pan American Health Organization. Renewing primary health care in the Americas: a position paper of the Pan American Health Organization/World Health Organization (PAHO/WHO). Washington, D.C: Pan American Health Organization; 2007.

6. Centro de Políticas Públicas UC. Fortalecimiento de la atención primaria de salud: propuestas para mejorar el sistema sanitario chileno [Internet]. 2014 [citado el 25 de diciembre de 2017]. Disponible en: http:// politicaspublicas.uc.cl/wp-content/uploads/2015/02/ fortalecimiento-de-la-atencion-primaria-de-salud-propuestas-para-mejorar-el-sistema-sanitario.pdf

7. Ministerio de Salud, Subsecretaría de Redes Asistenciales, División de Gestión de la Red Asistencial. Modelo de Atención Intergral en Salud [Internet]. 2005. Disponible en: http://www.bibliotecaminsal.cl/wp/wp-content/ uploads/2016/03/1.pdf

8. Blozik E, Reich O, Rapold R, Scherer M, Djalali S, Huber $\mathrm{F}$, et al. Evidence-based indicators for the measurement of quality of primary care using health insurance claims data in Switzerland: results of a pragmatic consensus process. BMC Health Serv Res 2018; 18 (1): 743.

9. de Cruppé W, Kleudgen S, Diel F, Burgdorf F, Geraedts M. Feasibility of 48 quality indicators in ambulatory care in Germany: a cross-sectional observational study. Z Evid Fortbild Qual Gesundhwes 2015; 109 (9): 682-94.

10. Australian Commission on Safety and Quality in Health Care. Draft national set of practice-level indicators of safety and quality for primary health care [Internet]. 2012. Disponible en: https://www.safetyandquality.gov. au/wp-content/uploads/2012/02/Draft-national-practice-level-indicators-of-safety-and-quality-for-primary-health-care.pdf.

11. Ministerio de Salud, Subsecretaría de Redes Asistenciales, División de Atención Primaria. Orientaciones para la Implementacion del Modelo de Atencion Integral de Salud Familiar y Comunitaria [Internet]. Disponible en: https://www.minsal.cl/portal/url/item/e7b24eef3e5cb5d1e0400101650128e9.pdf.

12. Pública MDS Subsecretaría de Salud. Ley-20645 15Dic-2012 Ministerio De Salud, Subsecretaría De Salud Pública [Internet]. Ley Chile - Biblioteca del Congreso Nacional. 2012 [citado el 8 de julio de 2018]. Disponible en: https://www.leychile.cl/Navegar?idNorma=1046749.

13. Braddock $\mathrm{CH}$, Snyder L, for the American College of Physicians Ethics and Human Rights Committee, Ethics and Time, Time Perception, and the Patient-Physician Relationship. Philadelphia: American College of Physicians; 2005: Position Paper. (Available from American College og Physicians, 190 N. Independence Mall West, Philadelphia, PA 19106).

14. Braddock $\mathrm{CH}$, Snyder L. The Doctor Will See You Shortly. J Gen Intern Med 2005; 20 (11): 1057-62.

15. Campbell SM, Hann M, Hacker J, Burns C, Oliver D, Thapar A, et al. Identifying predictors of high quality care in English general practice: observational study. BMJ 2001; 323 (7316): 784.

16. Rosser WW, Colwill JM, Kasperski J, Wilson L. Progress of Ontario's Family Health Team Model: A Patient-Centered Medical Home. Ann Fam Med 2011; 9 (2): 165-71.

17. Norful A, Martsolf G, deJacq K, Poghosyan L. Utilization of Registered Nurses in Primary Care Teams: A Systematic Review. Int J Nurs Stud 2017; 74: 15-23.

18. Freund T, Peters-Klimm F, Boyd CM, Mahler C, Gensichen J, Erler A, et al. Medical Assistant-Based Care Management for High-Risk Patients in Small Primary Care Practices: A Cluster Randomized Clinical Trial. Ann Intern Med 2016; 164 (5): 323-30.

19. Ferrer RL, Mody-Bailey P, Jaén CR, Gott S, Araujo S. A medical assistant-based program to promote healthy behaviors in primary care. Ann Fam Med 2009; 7 (6): 504-12.

20. Adewale V, Anthony D, Borkan J. Medical assistants' roles in electronic health record processes in primary care practices: the untold story. J Med Pract Manag MPM 2014; 30 (3): 190-6. 
21. Anderson A, Proudfoot JG, Harris M. Medical assistants: a primary care workforce solution? Aust Fam Physician 2009; 38 (8): 623-6.

22. Bodenheimer T, Laing BY. The Teamlet Model of Primary Care. Ann Fam Med 2007; 5 (5): 457-61.

23. Bodenheimer T, California HealthCare Foundation. Building teams in primary care: 15 case studies [Internet]. Oakland, Calif.: California HealthCare Foundation; 2007 [citado 9 de julio de 2018]. Disponible en: http://bibpurl.oclc.org/web/19255 http://www.chcf.org/ documents/chronicdisease/BuildingTeamsInPrimaryCareCaseStudies.pdf.

24. Ministerio de Salud, División Jurídica. Aprueba norma técnica administrativa que regula la relación asistencial docente y establece proceso de asignación de campo clínico docente de formación profesional y técnica de pregrado [Internet]. 2017. Disponible en: https://www. minsal.cl/wp-content/uploads/2017/09/DS-N\%C2\%BA19.aprueba-NGTA-RAD.05-09-17.pdf.

25. Municipalidad de Maipú. Capítulo 6 Maipú Sano: Gestión en Salud Municipal. En: Cuenta Pública de Gestión Municipal Maipú 2017 [Internet]. 2018. Disponible en: http:// www.transparenciamaipu.cl/wp-content/uploads/2018/04/ Cap_6_Gestion_en_Salud_Municipal.pdf.

26. Cartografía Base Comunal. Centros de Salud Municipal y Pública [Internet]. 2017. Disponible en: http://sitma. maps.arcgis.com/apps/MapAndAppGallery/index.html?appid=97f27800760e4147b0753f353fa7eda4.

27. Escuela de Salud Pública. Universidad de Chile. Estudio para la adaptación y validación de instrumento de medición del trato al usuario en los establecimientos de Atención Primaria Municipal de la Red Pública de Salud: Informe Final. Santiago: Escuela de Salud Pública; [no impreso] 2012. [Internet]. Disponible en: http:// utransparente.med.uchile.cl/2014/Terceros/12\%20 2014\%20r3847\%20Resol\%20aprueba\%20acuerdo\%20 FEN\%20FMUCH\%20trato\%20usuario\%20Minsal\%20 APS.pdf.

28. Escuela de Salud Pública de la Universidad, de Chile. Estudio Nacional de Evaluación de Percepción del Trato a Usuarios en Establecimientos de Salud Municipal del Sistema Público de Salud Informe Final de Resultados [Internet]. 2013. Disponible en: http://www. ssmso.cl/tmpArchivos/comunicaciones/2014_07_07/ Encuesta\%20Nacional\%20Bono\%20Trato\%20Usuario_APS_2013.pdf.

29. Prislin MD, Morrison E, Giglio M, Truong P, Radecki S. Patients' perceptions of medical students in a longitudinal family medicine clerkship. Fam Med 2001; 33 (3): 187-91.

30. Beca I JP, Browne L F, Valdebenito H C, Bataszew V A, Martínez I MJ. Relación estudiante-enfermo: Visión del paciente. Rev Med Chile 2006; 134 (8): 955-9.

31. Saba GW, Villela TJ, Chen E, Hammer H, Bodenheimer T. The myth of the lone physician: toward a collaborative alternative. Ann Fam Med 2012; 10 (2): 169-73.

32. Green LV, Savin S, Lu Y. Primary Care Physician Shortages Could Be Eliminated Through Use Of Teams, Nonphysicians, And Electronic Communication. Health Aff (Millwood) 2013; 32 (1): 11-9.

33. Weller J, Boyd M, Cumin D. Teams, tribes and patient safety: overcoming barriers to effective teamwork in healthcare. Postgrad Med J 2014; 90 (1061): 149-54.

34. Schottenfeld L, Petersen D, Peikes D, Ricciardi R, Burak $\mathrm{H}$, McNellis R, Genevro J. Creating Patient-Centered Team-Based Primary Care. AHRQ Pub. No. 16-0002EF. Rockville, MD: Agency for Healthcare Research and Quality. March 2016; 27.

35. Pontificia Universidad Católica de Chile. Escuela de Medicina. Dirección de Postgrado. Departamento de Medicina Familiar. Programas de Especialidad Médica en Medicina Familiar [Internet]. 2018. Disponible en: https://medicina.uc.cl/wp-content/uploads/2018/11/ Programa-Medicina-Familiar-Adulto2018.pdf.

36. Universidad de Chile. Programa de Título Profesional de Especialista en Medicina General Familiar [Internet]. Disponible en: http://www.uchile.cl/postgrados/9984/ medicina-general-familiar.

37. Parada-Lezcano M, Romero S MI, Moraga Cortés F. Educación médica para la Atención Primaria de Salud: visión de los docentes y estudiantes. Rev Med Chile 2016; 144 (8): 1059-66.

38. How can experience in clinical and community settings contribute to early medical education? A BEME systematic review: Medical Teacher: Vol 28, No 1 [Internet]. [citado el 12 de febrero de 2019]. Disponible en: https://www.tandfonline.com/doi/ abs/10.1080/01421590500410971?journalCode=imte20.

39. Family Medicine Clerkship [Internet]. [citado $11 \mathrm{de}$ febrero de 2019]. Disponible en: https://familymedicine. georgetown.edu/divisions/medicalstudenteducationdivision/courses/fammedclerkship.

40. Family Medicine Clerkship [Internet]. [citado 11 de febrero de 2019]. Disponible en: https://medicine.mercer. edu/academics/curriculum/fm-clerkship/

41. Third Year Clerkship» Family Medicine | Boston University [Internet]. [citado 11 de febrero de 2019]. Disponible en: https://www.bu.edu/familymed/medical-student-ed/third-year/

42. Ministerio de Desarrollo Social. Síntesis de Resultados CASEN 2017 [Internet]. 2018. Disponible en: http:// observatorio.ministeriodesarrollosocial.gob.cl/casen-multidimensional/casen/docs/Resultados_Salud_casen_2017.pdf. 